\title{
Parameter Calibration of Xin'anjiang Model Based on Complex Genetic Algorithm
}

\author{
ZHOU Yujia", a, CHEN Yifan ${ }^{1, ~ b}$, DAN Jiaojiao ${ }^{1}$, LIU Lijun ${ }^{1}$ \\ ${ }^{1}$ Key Laboratory of Disaster Prevention and Reduction, Zhejiang Institute of Hydraulics \\ \& Estuary, Hangzhou 310020, China \\ azhouyujia77@126.com, bzjucoolboy@163.com
}

\begin{abstract}
Keywords: complex genetic algorithm; Xin'anjiang model; parameter calibration Abstract: Traditional manual parameter debugging is subjective and requires professional experience. To make up for the deficiencies, a new kind of parameter debugging approach based on complex genetic algorithm is proposed to calibrate the parameters of Xin'anjiang model. The proposed approach combines genetic algorithm of global optimization with complex method of local optimization. This paper verify the performance of the complex genetic algorithm by using the stratification calibration method with an example of watershed of Xiahuitou hydrometric station in Zhejiang Province. The results show that the difference between the multi-year average runoff and simulated runoff is $1 \%$, and the annual runoff process reaches grade $\mathrm{B}$ standard or above.
\end{abstract}

\section{Introduction}

Flood forecasting is an indispensable work that serves national economy. It helps people effectively prevent floods, reduce flood losses, and better control and use of water resources. It's an important non-engineering measure for flood control and disaster reduction[1]. Xin'anjiang model is widely used in runoff calculation and flood forecasting in wet and humid areas of southern China. Most of Xin'anjiang model parameters have clear physical meanings, which has a great influence on rationality and accuracy of model calculation results.

In recent years, with the development of computer technology and its broad applications, parameter automatic calibration methods have become important tools to avoid subjectivity and randomness in artificial parameter calibration. Since Xin'anjiang model has been put forward in the 1970s, automatic optimization methods, such as simulated annealing, SCE-UA, particle swarm optimization, genetic algorithm [2-5] have successively applied to the parameters optimization of Xin'anjiang model. Genetic algorithm is frequently used to solve the non-analytic objective function and constraint problem with its simple generalization, robustness and global optimization features, and becomes an important approch to study the parameter calibration[6]. However, the genetic algorithm has low computational efficiency and is easy to fall into the local optimum when the search space is large. To solve this problem, many researchers tried to adopt a hybrid strategy to achieve the mutual complementarity. In order to make up for the shortcomings of traditional genetic algorithm, $\mathrm{Li}$ shuiyan proposed an improved genetic algorithm to solve the problem of parameter calibration in Xin'anjiang model[7]. Meng Xinhua et al. applied a hybrid algorithm combining simulated annealing algorithm with traditional genetic algorithm in the parameter selection of Xin'anjiang model[8].

This paper focuses on the parameter calibration of Xin'anjiang model, and proposes a complex genetic algorithm that combines genetic algorithm with complex method.

\section{Complex genetic algorithm}

\section{Complex Method}

Complex method is an effective solution to the problem of nonlinear programming constraints[9,10]. Firstly, it selects K vertices as vertices of an initial complex in the optimal space, then calculates and compares the value of each vertex objective function. secondly, the vertex with maximum value is removed as the worst vertex and replaced by a new vertex through mapping calculation. Then a new complex is constituted. It needs to repeat the above process until convergence. 
Finally, the vertex with minimum objective function value in the last complex is taken as the approximate optimal solution. The details of complex method are shown as follows:

Step 1: Construct an initial complex;

Step 2: For each vertex $j=1,2, \ldots, K$, calculate the objective function values $f\left(X^{(j)}\right)$. Then choose the best vertex $X^{(L)}$ and the worst vertex $X^{(H)}$;

Step 3: Calculate the centroid of the complex $X_{0}$ ( without the worst vertex $X^{(H)}$ );

Step 4: Calculate the mapping vertex $X^{(R)}$;

$X^{(R)}=X_{0}+\alpha\left(X_{0}-X^{(H)}\right)$

where $\alpha$ is the mapping coefficient. In general, $\alpha=1.3$. It needs to check whether $X^{(R)}$ is in the feasible region. Otherwise, calculating the mapping vertex with half mapping coefficient according equation (1) until $X^{(R)}$ enters into the feasible region.

Step 5: Construct a new complex;

The objective function value of the mapping vertex is calculated and compared with the value of the worst vertex. In general, it may have two different results:

1) The mapping vertex is better than the worst vertex, i.e., $f\left(X^{(R)}\right)<f\left(X^{(H)}\right)$

In this case, adopt $X^{(R)}$ instead of $X^{(H)}$ to form a new complex.

2) The mapping vertex is worse than the worst vertex, i.e.,

$$
f\left(X^{(R)}\right)>f\left(X^{(H)}\right)
$$

In this case, the mapping vertex can be moved closer by using half the mapping coefficient $\alpha$. If $f\left(X^{(R)}\right)<f\left(X^{(H)}\right)$ after decreasing $\alpha$, the result converts into the first result. If $\alpha$ is halved by many times, and less than a small positive $\delta$ that is predetermined, the mapping vertex is still not better than the worst vertex, then change the mapping direction using the second worst vertex $X^{(\delta H)}$ by the same method until a new complex is constructed.

If the result satisfies the iteration termination condition, take $X^{(L)}$ and $f\left(X^{(L)}\right)$ in the last complex as the optimal solution, then stop. Otherwise, reture to Step 2.

\section{Combination of complex method and genetic algorithm}

Genetic algorithm has good global optimization feature, but may fall into local optimal and premature convergence. Complex method has good local optimization feature, but can fail in iterative calculation, especially in the complex optimization problem and it is also great affected by the initial complex $[11,12]$. Considering the advantages and disadvantages of the two algorithms, the combined algorithm is obtained. Firstly, using the genetic algorithm for global optimization and getting a better solution space. Then an initial complex is constructed in the better solution space. Finally, global optimal solution is obtained through the quadratic optimization by complex method. Figure 1 shows the process of complex genetic algorithm. In this paper, it assumes the chromosome compilation is carried out by binary coding. The initialization is performed before calculated, including setting the maximum generation number Tmax, population size, crossover rate, mutation rate and initializing individual. The objective function value is the deviation between measured data and simulated data. The constraint conditions are in reasonable range for each parameter. 


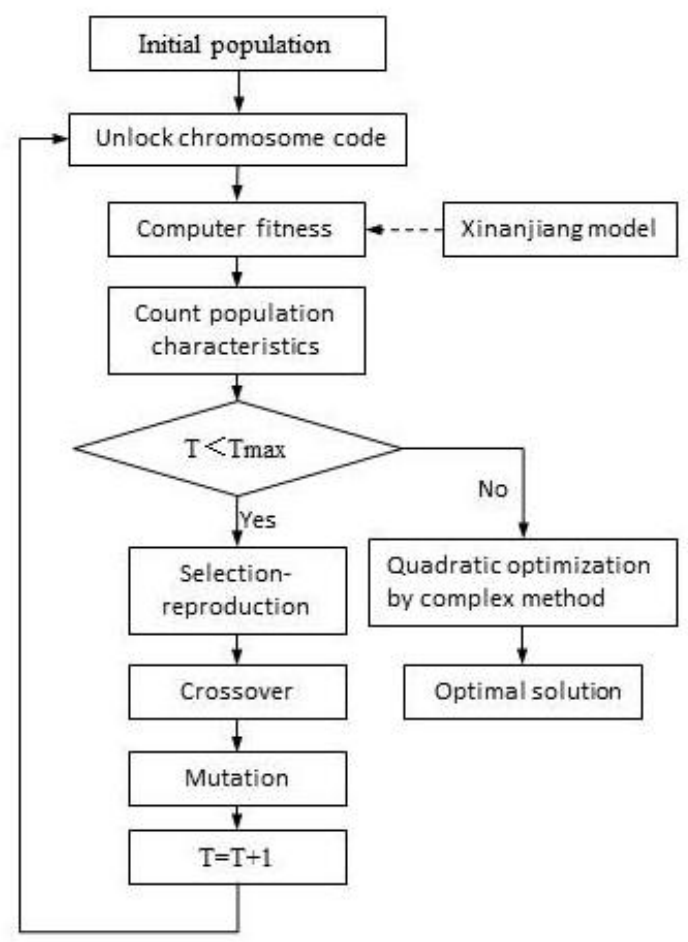

Fig. 1 Process of complex genetic algorithm

\section{Complex genetic algorithm and Xin'anjiang model}

Xin'anjiang model can forecast the runoff of watershed according to the input of precipitation and potential evapotranspiration[13]. The structure process and parameter physical meanings of Xin'anjiang model are shown in figure 2 and table 1.

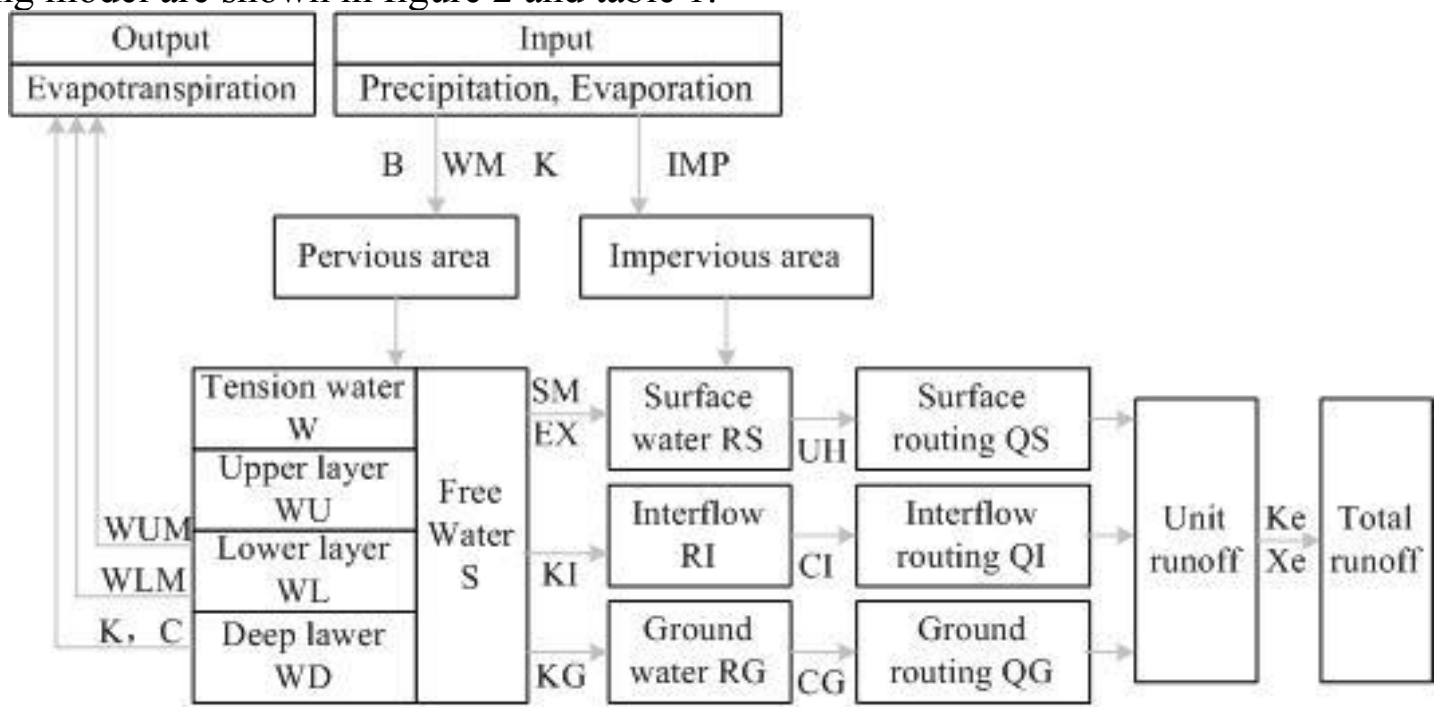

Fig.2 Structural process of Xin'anjiang model 
Table.1 Parameter physical meanings of Xin'anjiang model

\begin{tabular}{|c|c|c|c|}
\hline Classification & Parameter & Physical Meaning & Normal Value Range \\
\hline \multirow{16}{*}{ Main Parameters } & $W M / \mathrm{mm}$ & average tension water capacity of the watershed & $80 \sim 200$ \\
\hline & $W U M / \mathrm{mm}$ & tension water capacity of upper layer & $5 \sim 20$ \\
\hline & $W L M / \mathrm{mm}$ & tension water capacity of lower layer & $60 \sim 90$ \\
\hline & $W D M / \mathrm{mm}$ & tension water capacity of deep layer & \\
\hline & K & evapotranspiration coefficience & $0.1 \sim 1.0$ \\
\hline & $C$ & evapotranspiration coefficience of deep layer & $0.1 \sim 0.2$ \\
\hline & $B$ & tension water capacity curve & $0.2 \sim 0.3$ \\
\hline & $I M P$ & the impermeable area of the basin area ratio & $0.001 \sim 0.02$ \\
\hline & $S M / \mathrm{mm}$ & free water capacity of topsoil & $5 \sim 45$ \\
\hline & $E X$ & curve of free water capacity of topsoil & $1.0 \sim 1.5$ \\
\hline & $K I$ & free water outflow coefficient of topsoil to & \\
\hline & $K G$ & $\begin{array}{l}\text { 1nterflow } \\
\text { free water outflow coefficient of topsoil to } \\
\text { groundwater }\end{array}$ & $0<K I+K G<1$ \\
\hline & $C I$ & regression coefficient of interflow & $0.3 \sim 0.8$ \\
\hline & $C G$ & regression coefficient of groundwater & $0.98 \sim 0.998$ \\
\hline & $N K$ & linear reservoir number & $1.0 \sim 5.0$ \\
\hline & $N T$ & concentration time & $2.0 \sim 10$ \\
\hline \multirow{4}{*}{$\begin{array}{l}\text { Initial condition of } \\
\text { parameters }\end{array}$} & WU/mm & tension water storage of upper layer & \\
\hline & $W L / \mathrm{mm}$ & tension water storage of lower layer & \\
\hline & $W D / \mathrm{mm} \backslash$ & tension water storage of deep layer & \\
\hline & $S / \mathrm{mm}$ & free water storage of topsoil & \\
\hline
\end{tabular}

Xin'anjiang model can be divided into four coupling calculation processes, namely evaporation, runoff, water partitioning, and flow concentration. The parameters are also divided into four independent categories, respectively. The stratification calibration method by controlling the objective function is used to get accurate and reasonable optimization parameters[14].

(1) Evaporation and runoff processes. The parameters of evaporation and runoff processes are $K 、 C 、 B 、 I M P, W U M, W L M$ and $W D M$. These parameters determine the total runoff and control the water balance between rainfall, evaporation and runoff. As the rainfall is known, the parameters can be optimized by the water balance equation. With the measured runoff data, it only needs to minimize the error of the multi-annual flow. Formula is as follow:

$$
\Delta Q=\sum_{i=1}^{n} A B S\left[Q_{\text {obs }}(i)-Q_{\text {sim }}(i)\right], \quad i=1,2, \cdots, n
$$

where $Q_{\text {obs }}(i)$ is the measured flow, $\mathrm{m}^{3} / \mathrm{s}, Q_{\text {sim }}(i)$ is the calculated flow, $\mathrm{m}^{3} / \mathrm{s}, \quad n$ is the data series length, respectively.

(2) Water partitioning and flow concentration processes. The parameters of water partitioning and flow concentration processes are $S M, E X, K I, K G 、 C I, C G 、 N K 、 N T, K E$, and $X E$. These parameters determine the runoff process. The ground runoff affects the high water process, and the main influencing parameters are $S M, E X, K I, K G, N K$, and $N T$. The underground runoff affects the low-water process, and the main influencing parameters are $K I, K G$ and $C G$. Parameter $S M$ also has a certain impact.

The objective function $F_{L O G}$ is selected to calibrate the parameters $S M, K I, K G, C G, N K$ and $N T$. As parameter $E X$ has a stable value between 1 and 1.5, it does not need to consider it for optimization. The formula is as follow:

$$
F_{L O G}=\frac{\sum_{i=1}^{n} A B S\left\{L O G\left[\frac{Q_{o b s}(i)}{Q_{\text {sim }}(i)}\right]\right\}}{\sum_{i=1}^{n} L O G\left[Q_{\text {obs }}(i)\right]}, i=1,2, \cdots, n
$$

$S M$ is sensitive to the high water process, using the objective function $F_{A B S}$ to optimize $S M$. The formula is as follow: 


$$
F_{A B S}=\frac{\sum_{i=1}^{n} A B S\left[Q_{o b s}(i)-Q_{\text {sim }}(i)\right]}{\sum_{i=1}^{n} Q_{o b s}(i)}, i=1,2, \cdots, n
$$

$N K, N T$ have great influence on Nash-Sutcliffe efficiency coefficient, selecting the objective function $F_{N A S H}$ to optimize $N K, N T$. The formula is as follow:

$$
F_{N A S H}=1-\frac{\sum_{i=1}^{n}\left[Q_{o b s}(i)-Q_{\text {sim }}(i)\right]^{2}}{\sum_{i=1}^{n}\left[Q_{o b s}(i)-\overline{Q_{o b s}}\right]^{2}}, i=1,2, \cdots, n
$$

where $\overline{Q_{o b s}}$ is the measured average flow, $\mathrm{m}^{3} / \mathrm{s}$.

The stratification calibration method can make the value of each parameter more consistent with its physical significance in Xin'anjiang model. It is helpful to reduce the total runoff error and make the flow process fit better. After the model parameters are stratified, the complex genetic algorithm is used to optimize the model.

\begin{tabular}{|c|c|c|c|c|}
\hline Algorithm & Parameter & Significance & Value range & $\begin{array}{l}\text { Value of this } \\
\text { paper }\end{array}$ \\
\hline \multirow{5}{*}{$\begin{array}{l}\text { Genetic } \\
\text { algorithm }\end{array}$} & ga-numpara & number of optimization parameters & & \\
\hline & popsize & population size & $60-200$ & 100 \\
\hline & maxgen & maximum generation number & $60-100$ & 80 \\
\hline & pcross & individual crossover rate & $0.8-1.0$ & 0.9 \\
\hline & pmutation & individual mutation rate & $0.001-0.02$ & 0.01 \\
\hline \multirow{3}{*}{$\begin{array}{c}\text { Complex } \\
\text { method }\end{array}$} & $N$ & dimension & & 3 \\
\hline & $K$ & number of vertex & $\mathrm{N}+1 \leq \mathrm{K} \leq 2 \mathrm{~N}$ & 5 \\
\hline & $a$ & mapping coefficient & 1.3 & 1.3 \\
\hline
\end{tabular}

Tab.2 Parameter values of genetic algorithm and complex method

\section{Case study}

\section{Study area and data}

Zhuxi River is a major tributary of Yong'anxi River. It originates from Xiakeng in the southeast of Xianju and flows from south to north through Fangshan village, Mei'ao, Xiahuitou, Dazhan, finally flows into Yong'an Stream near Houlincun village. Zhuxi River is $49.2 \mathrm{~km}$ long and has a watershed of $379.3 \mathrm{~km}^{2}$. Xiahuitou hydrologic station locates below Zhuxi River with a watershed of $241 \mathrm{~km}^{2}$. The topography of Zhuxi watershed is dominated by the middle and low mountains and appears with large river slope, urgent flow and steep flood process. However, Zhuxi watershed has good vegetation with less soil and water loss.

Xiahuitou hydrologic station locates in Shangma village, built in 1956. There are 4 precipitation stations named Miaoliao, Xishang, Xianjumeiao and Dahong in watershed. This paper use the data from 1972 to 1981 as calibration period, the data from 1982 to 1988 as verification period. Because of the data length sequence requirement, it adopts precipitation data from Xiahuitou, Miaoliao, Xishang, and Dahong sations, evaporation data from Xianjumeiao station, daily runoff from Xiahuitou hydrologic station. 


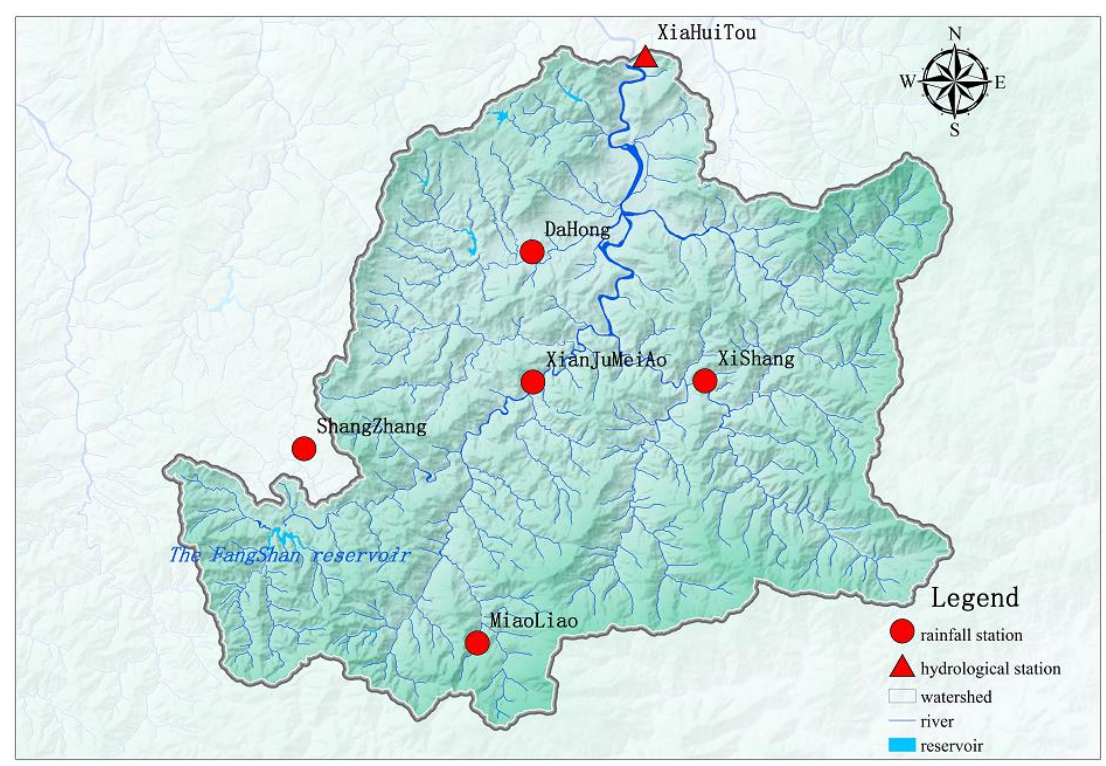

Fig.3 Site distribution of watershed above Xiahuitou hydrologic station

\section{Parameter calibration result}

When using genetic algorithm for optimization, it can properly set a wide range of sensitive parameters. It is helpful to find the optimal solution space, but will lead to slow down the convergence time. The parameters with weak sensitivity should be set in a reasonable range. The results of insensitive parameters are considered as the optimal solution by genetic algorithm optimization and not participate in complex method optimization. The results of parameter optimization are shown in table 3

Table 3 Optimization results with complex genetic algorithm

\begin{tabular}{cccc}
\hline Parameter & Initial value & Calibration results & Range \\
\hline$W M$ & 90 & 122 & $80 \sim 200$ \\
$W U M$ & 20 & 6 & $5 \sim 20$ \\
$W L M$ & 50 & 60 & $60 \sim 90$ \\
$W D M$ & 20 & 56 & \\
$K$ & 0.8 & 0.974 & $0.1 \sim 1.0$ \\
$C$ & 0.15 & 0.165 & $0.1 \sim 0.2$ \\
$B$ & 0.25 & 0.246 & $0.2 \sim 0.3$ \\
$I M P$ & 0.1 & 0.005 & $0.001 \sim 0.1$ \\
$S M$ & 15 & 14 & $5 \sim 45$ \\
$E X$ & 1.2 & 1.2 & $1.0 \sim 1.5$ \\
$K I$ & 0.4 & 0.23 & $0<K I+K G<1$ \\
$K G$ & 0.3 & 0.169 & \\
$C I$ & 0.5 & 0.399 & $0.3 \sim 0.8$ \\
$C G$ & 0.98 & 0.997 & $0.95 \sim 0.998$ \\
$N K$ & 1.5 & 4.731 & $1.0 \sim 5.0$ \\
$N T$ & 3 & 2.083 & $2.0 \sim 10$ \\
\hline
\end{tabular}

\section{Model verification}

Xin'anjiang model validation is conducted with the parameters from optimization results and the data from verification period. Firstly, the initial condition parameters of the model are adjusted so that the runoff process in the first year of the validation period is in good fittness with the measured runoff progress. Then it simulates other years. Secondly, we calculate the relative error of the annual runoff and the deterministic coefficient of runoff process. Finally, it evaluates the accuracy of the forecast. The validation results are shown in table 4. 
Table 4 Runoff error of watershed above Xiahuitou hydrologic station

\begin{tabular}{ccccc}
\hline Year & Measured runoff/mm & Simulated runoff/mm & Relative error & Deterministic coefficient \\
\hline 1982 & 1076.6 & 1104.7 & $3 \%$ & 0.74 \\
1983 & 1071.2 & 1104.5 & $3 \%$ & 0.75 \\
1984 & 1112.0 & 1045.3 & $6 \%$ & 0.73 \\
1985 & 1055.8 & 998.6 & $5 \%$ & 0.89 \\
1986 & 708.3 & 700.6 & $1 \%$ & 0.70 \\
1987 & 1332.1 & 1310.5 & $2 \%$ & 0.93 \\
1988 & 909.0 & 904.2 & $1 \%$ & 0.86 \\
average annual & 1037.9 & 1024.1 & $1 \%$ & \\
runoff & & & & \\
\hline
\end{tabular}

In table 4, the average annual simulated runoff is $1024.1 \mathrm{~mm}$, and the average annual measured runoff is $1037.9 \mathrm{~mm}$. There is an absolute error of $13.8 \mathrm{~mm}$ and a relative error of $1 \%$. The annual runoff volume errors are all within $10 \%$. The annual deterministic coefficients are all above 0.7 . According to the standard for hydrological information and hydrological forecast issued by China, the accuracy of the annual simulated runoff reaches the grade B standard or above.

\section{Conclusion}

This paper proposed a complex genetic algorithm to calibrate parameters of Xin'anjiang model. One case study in Zhuxi watershed is given to indicate that the proposed algorithm can be feasible for optimizing parameters of Xin'anjiang model and can achieve good results.

\section{Acknowledgements}

This work was financially supported by the National Natural Science Foundation of China (51609213), and the Science and Technology Foundation of Zhejiang Institute of Hydraulics and Estuary(ZIHE2016008).

\section{References}

[1] Li Longling, Ren Jinzheng. Features of flood disaster zoning in China[J]. CHINA WATER RESOURCES, 2014, (07):48-51.

[2] Zhang Xinnan. Application of Fuzzy Mathematies to Automatie Calibration of Xinganjiang Model Parameters [J]. JOURNALOFHOHAIUNIVERSITY, 1988, 03:128-135.

[3] SHEN Tonglin, JIANG Tiebing, FAN Wentao. Using Parallel Genetic Algorithm to Calibrate Conceptual Rainfall-Runoff Model[A].Systems engineering society of China. Systems Engineering, Systems Science and Complexity Research-Proceeding of 11th Annual Conference of Systems Engineering Society of China[C]. Systems engineering society of China, 2000:6.

[4] Hapuarachchi H.A.P., LI Zhijia, WANG Shouhui. Application of SCE-UA Method for Calibrating the Xinanjiang Watershed Model[J]. JOURNAL OF LAKE SCIENCES, 2001, 04: 304-314.

[5] DONG Jieping, LI Zhijia, DAI Jiannan. Application of SCE-UA algorithm to optimization of Xin'anjiang model parameters[J]. Journal of Hohai University, 2012, 05:485-490.

[6] WU Xin-yu, CHENG Chun-tian, ZHAO Ming-yan. Parameter calibration of Xinanjiang rainfall-runoff model by using parallel genetic algorithm[J]. SHUILI XUEBAO, 2004, 11:85-90.

[7] LI Shuiyan. An Improved Genetic Algorithm and its Application in the Rainfall-runoff Model[D]. Hohai University,2006.

[8] MENG Xinhua, TU Qiyu, ZHOU Nianhua, ZHENG Nan. Application of Hybrid Simulated Annealing Genetic Algorithm to Parameter Estimation of Xinanjiang Model [J]. Hydropower Automation and Dam Monitoring, 2009, 03:64-67.

[9] Siren Wang. The Introduction of the Complex Method and its application[J]. JIANGXI HYDRAULIC SCIENCE \& TECHNOLOGY, 1995, 02:93-97. 
[10] CHEN Gang, ZHANG Lin, CHEN Jian-kang, HE Xian-song. Application of the complex method for reliability analysis of arch dams [J]. SHUILI XUEBAO, 2003, 02:98-101,106.

[11] LI Liang, CHI Shichun, LIN Gao. The complex method based on ant colony algorithm and its application to the slope stability analysis [J]. Chinese Journal of Geotechnical Engineering, 2004, 05:691-696.

[12] Shanshan Cui. Some Improvements of the Genetic Algorithm and their Applications[D]. University of Science and Technology of China,2010.

[13] Zhao Renjun. Watershed hydrological simulation: Xin'anjiang Model and Shanbei Model[M]. Beijing: Water Resources and Hydropower Press, 1984.

[14]) CHEN Jiongfeng, ZHANG Wanchang. Application of Genetic Algorithm for Model Parameter Calibration in Daily Rainfall-Runoff simulations with the Xinanjiang Model[J]. JOURNAL OF CHINA HYDROLOGY, 2006, (04):32-38. 